\title{
EBM-Reform zum 01.04.2020
}

\author{
Christoph Sucker $^{1}$ Günther Kappert ${ }^{2}$ Jürgen Koscielny ${ }^{3}$
}

1 Medizinisches Versorgungszentrum (MVZ) COAGUMED
Gerinnungszentrum, Berlin, Germany
2 Gerinnungszentrum Rhein-Ruhr, Duisburg, Germany
${ }^{3}$ Gerinnungsambulanz mit Hämophiliezentrum im ambulanten
Gesundheitszentrum (AGZ), Charité, Universitätsmedizin Berlin,
Berlin, Germany

Hämostaseologie 2020;40:384-385.

Nach jahrelangen Verhandlungen ist am 01.04.2020 der Neue Einheitliche Bewertungsmaßstab (EBM) für die Abrechnung kassenärztlich Versicherter in Kraft getreten. Aufbau und Struktur des EBM blieben unverändert. Es handelte sich um eine „kleine“ EBM-Reform, wobei die Leistungen überprüft und an die aktuelle Kostenstruktur angepasst wurden. Hierbei wird von einem kalkulatorischen Arztlohn von 117.000 Euro pro Jahr ausgegangen. Es wurden nur wenige Leistungen neu in den EBM aufgenommen und die Honorierung insgesamt kaum verändert; dies ist dem Gebot der Kostenneutralität geschuldet, so dass die Gesamtausgaben der Kostenträger durch Abrechnung der EBM-Leistungen konstant bleiben. Diese Ausgabenneutralität wurde vielfach kritisiert und wird aus Sicht des Berufsverbandes den wachsenden Anforderungen bei der Versorgung von Patienten nicht gerecht.

Grundsätzlich wurden in der neuen Fassung des EBM technische Leistungen abgewertet und Gesprächsleistungen aufgewertet. Große Umverteilungen haben nicht stattgefunden. Seitens des Vorstandes der Kassenärztlichen Bundesvereinigung (KBV) wurde betont, dass weitere EBM-Reformen nur bei Anhebung des finanziellen Rahmens bzw. Wegfall der Kostenneutralität unterstützt werden. Hier bleibt die weitere Entwicklung abzuwarten.

Wichtig ist im hämostaseologischen Bereich die Bewertung der Labordiagnostik. Zur Erbringung dieser Leistungen ist eine Abrechnungsgenehmigung durch die zuständige kassenärztliche Vereinigung erforderlich. In der Neufassung des EBM ist es erfreulicherweise nicht zu einer Abwertung der Abrechnung für Laborleistungen nach den Kapiteln 11,12, 19 und 32 EBM gekommen. Ein genauerer Blick auf die hämostaseologische Labordiagnostik in Kapitel 32.2.4 und 32.3.3 EBM zeigt folgende Abrechnungsziffern und Honorierungen:

Der Vergleich mit der Vorversion des EBM zeigt, dass die Bewertung sämtlicher genannter Laborleistungen unverändert ist. Zu beklagen ist die weiterhin schlechte Honorierung von Basistests der Gerinnungsdiagnostik wie aPTT (32112), TPZ (32113), TZ (32115) und Fibrinogen (32115); dies Leis-
Address for correspondence Priv.-Doz. Dr. med. habil. Christoph Sucker, COAGUMED Gerinnungszentrum GmbH, Medizinisches Versorgungszentrum (MVZ), Tauentzienstraße 7b/c, 10789 Berlin (e-mail: cs@coagumed.de).

tungen sind insbesondere für Gerinnungspraxen mit eigenem Gerinnungslabor kaum kostendeckend zu erbringen.

In die Neufassung des EBM wurden für die hämostaseologischen Laboruntersuchungen keine neuen Abrechnungsziffern aufgenommen. Eine Abrechnung sonstiger Gerinnungsuntersuchungen kann ggf. über die EBM-Ziffern 32208 und 32227 für „ähnliche Untersuchungen“ erfolgen; es wird darauf hingewiesen, dass die Abrechnung von Untersuchungsverfahren nach diesen Ziffern je nach KV-Bereich gesondert genehmigungspflichtig ist.

\section{Mustervertrag zur ärztlichen Versorgung nach § 132 i SGBV mit dem Verband der Ersatzkassen (VDEK)}

Den Vorständen des Berufsverbandes der Deutschen Hämostaseologen (BDDH) und der Gesellschaft für Thrombose und Hämostaseforschung (GTH) ist es trotz den Widrigkeiten aufgrund der Corona-Pandemie gelungen, vor der Änderung des Vertriebsweges für Gerinnungsfaktorenpräparationen entsprechend dem Gesetz für mehr Sicherheit in der Arzneimittelversorgung (GSAV) den ersten Mustervertrag zur ärztlichen Versorgung nach $§ 132$ i Sozialgesetzbuch (SGB) V mit dem VDEK zu finalisieren. Dieser Vertrag kann ab sofort geschlossen werden (siehe auf der BDDH-Website: https:// bddh.org/aktuelles). Für neue Vertragspartner von Seiten der ärztlichen Therapeuten gilt das Vertragsabschlussdatum als Beginn, für die anderen mit bereits bestehenden Verträgen nach § 47.2 AMG wird grundsätzlich der 01.09.2020 als Vertragsbeginn avisiert. Weitere Musterverträge werden derzeit von den Vorständen des BDDH und der GTH verhandelt und hoffentlich bald finalisiert.

Da der 01.09.2020 wohl das endgültige Stichdatum für die Vertriebswegänderung werden wird, ist der neue Mustervertrag ein ärztlicher Versorgungsvertrag nach § 132 i SGBV und kein „Arzneimittelvertrag“ mehr. Der Vertrieb muss dann grundsätzlich über Apotheken erfolgen. Dies kann über öffentliche Apotheken oder Klinikapotheken, auch (c) 2020 Georg Thieme Verlag KG Stuttgart · New York
DOI https://doi.org/ 10.1055/a-1173-0610. ISSN 0720-9355. 
universitär, erfolgen, insbesondere im Rahmen der Notfallversorgung vertraglich nach § 43.3 AMG (Arzneimittelgesetz). Auch die Regelversorgung wird über öffentliche Apotheken und/oder Klinikapotheken erfolgen. Daher sollte jeder ärztlicher Therapeut im Vorfeld prüfen, wer als möglicher Partner auf Apothekenseite, z.B. für die Notfallversorgung, in Betracht kommt.

Da durch das GSAV auch die Patientenrechte gestärkt werden, weist der BDDH noch auf die Möglichkeit der Erteilung eines „Versorgungsauftrages für Hämophilie-Arzneimittel“ nach $\S 1$ Absatz 1 Apothekengesetz, $\S 17$ Absatz 2 ApBetrO und $\S 24$ ApBetrO im Rahmen der Regelversorgung ab 01.09.2020 durch die Patienten - sowohl für gesetzlich Versicherte (GKV) als auch für privatversicherte Patienten (PKV) - hin.

Des Weiteren wurde am 25.05.2020 der Verband der Hämophilie-Apotheken (VHA) mit Qualitätsmerkmalen gegründet. Damit beginnt eine „Qualitätsoffensive“ von
Seite der Apotheken. Die zuständige Website wird gerade aufgebaut.

Im Juni wird sich der Bundesverband für die ärztliche Spezialversorgung (BV ASV) und der Vorstand des BDDH mit Unterstützung der pädiatrischen GTH über praxisnähere Änderungen in der ASV der Hämophilie abstimmen und eine entsprechende Eingabe beim G-BA gemeinsam vorbereiten. Wir hoffen, dass eine praxisnähere Lösung erreicht wird. Dieser Vorgang wird- auch auf Grund der gesetzlichen Fristen - voraussichtlich Monate in Anspruch nehmen.

Für den Vorstand des Berufsverbandes der Deutschen Hämostaseologen e.V. (BDDH):

Priv.-Doz. Dr. Jürgen Koscielny, Berlin, Vorsitzender

Dr. Günther Kappert, Duisburg, Stellvertretender Vorsitzender

Priv.-Doz. Dr. Christoph Sucker, Berlin, Beisitzer des Vorstands

\begin{tabular}{|c|c|c|}
\hline EBM-Ziffer & Leistung & Vergütung \\
\hline 32110 & Blutungszeit (standardisiert) & 0,75 \\
\hline 32111 & Rekalzifizierungszeit & 0,75 \\
\hline 32112 & partielle Thromboplastinzeit (aPTT) & 0,60 \\
\hline 32113 & Thromboplastinzeit (TPZ) & 0,60 \\
\hline 32115 & Thrombinzeit (TZ) & 0,75 \\
\hline 32116 & Fibrinogenbestimmung & 0,75 \\
\hline 32203 & Thrombelastogramm & 16,60 \\
\hline 32205 & Reptilasezeit & 16,80 \\
\hline 32206 & APC-Resistenz & 15,60 \\
\hline 32207 & Lupusantikoagulans & 13,90 \\
\hline 32208 & ähnliche Untersuchungen & 19,20 \\
\hline 32210 & Antithrombin & 11,40 \\
\hline 32211 & Plasminogen & 18,30 \\
\hline 32212 & Fibrinmonomere, Fibrin(ogen)-Spaltprodukte (z.B. Dimere) & 17,80 \\
\hline 32213 & Faktor II & 18,80 \\
\hline 32214 & Faktor V & 18,40 \\
\hline 32215 & Faktor VII & 34,60 \\
\hline 32216 & Faktor VIII & 24,30 \\
\hline 32217 & Von-Willebrand-Faktor & 30,20 \\
\hline 32218 & Faktor IX & 24,10 \\
\hline 32219 & Faktor $\mathrm{X}$ & 29,10 \\
\hline 32220 & Faktor XI & 27,60 \\
\hline 32221 & Faktor XII & 27,60 \\
\hline 32222 & Faktor XIII & 25,90 \\
\hline 32223 & Protein C & 31,30 \\
\hline 32224 & Protein S & 31,30 \\
\hline 32225 & Plättchenfaktor 4 & 32,40 \\
\hline 32227 & ähnliche Untersuchungen & 20,70 \\
\hline 32228 & Thrombozytenfunktion & 33,20 \\
\hline 32229 & Von-Willebrand-Faktor-Multimeranalyse & 75,00 \\
\hline
\end{tabular}

